\title{
La Presencia de las mujeres en las Asociaciones de la Profesión Periodística en España
}

\author{
Francisco J. CARO GonZÁleZ \\ Universidad de Sevilla \\ fjcaro@us.es \\ María del Mar GARCÍA GORDILLO \\ Universidad de Sevilla \\ marggordillo@us.es
}

Recibido: 21 de marzo de 2011

Aceptado: 21 de diciembre de 2011

\begin{abstract}
Resumen
Las asociaciones de la prensa tienen por misión la defensa de la profesión periodística. En este trabajo analizamos estas instituciones desde la perspectiva de género, partiendo de la premisa de que una mayor presencia de mujeres en las mismas podría favorecer la igualdad en la profesión. Para ello estudiamos la composición de sus juntas directivas y presidencia según el sexo y comprobamos si reproducen o no los patrones que podemos encontrar en las empresas del sector. Las conclusiones a las que llegamos es que las mujeres están poco representadas en las juntas directivas, pero con un mayor equilibrio que en las empresas editoras, lo que nos hace atisbar una mejora en la igualdad en la profesión en un futuro próximo. Palabras clave: profesión periodística; asociaciones de la Prensa; género; igualdad laboral.
\end{abstract}

\section{The Presence of Women in the Associations of Journalists in Spain}

\begin{abstract}
Press associations have the task of defending the journalistic profession. In this paper we analyze these institutions from the perspective of gender, based on the premise that a greater presence of women in them could promote equality in the profession. To do so, we study the composition of their Boards of Directors and Chair by sex and verify if they reproduce the rules that we can find in the companies of the sector or not. The conclusion that we draw is that women are underrepresented on the Boards, but there is a better balance than in the press companies, which makes us discern an improvement in equality in the profession for the near future.
\end{abstract}

Keywords: journalism; press associations; gender; equality in the workplace.

Referencia normalizada: CARO RODRÍGUEZ, Francisco J. y GARCÍA GORDILLO, María del Mar (2012): "La Presencia de las mujeres en las Asociaciones de la Profesión Periodística en España". Estudios sobre el mensaje periodístico, vol. 18, núm. 2 (julio-diciembre), págs.: 689-701. Madrid, Servicio de Publicaciones de la Universidad Complutense.

Sumario: 1. Introducción. 2. El asociacionismo profesional en el periodismo. 3. Objetivos. 4. Metodología. 5. Resultados. 6. Conclusiones. 7. Referencias Bibliográficas.

\section{Introducción}

La relación de las mujeres como sujetos y objetos de la comunicación de masas es una realidad compleja en la que intervienen multitud de variables de diferente naturaleza. Esto ha sido puesto de manifiesto por el equipo de investigación SANIMUEI en varios artículos y trabajos presentados a congresos (CARO, GARCÍA, BEZUNARTEA y RODRÍGUEZ, 2008-2010), y por supuesto por el abultado número de investigaciones sobre el tema (DJERF-PIERRE, 2007; Burke, 2009; FARIAS, 2009; SORIANO, 2005; FrÖLICH, 2007; y, otros). 
Uno de los elementos que menos se ha investigado es el de la estructura de la profesión periodística y en qué medida ésta contribuye a la promoción de las mujeres en las empresas de comunicación.

Entre las barreras que más se citan, relacionadas con el "techo de cristal", aparece el hecho de que los hombres gozan de cierto "corporativismo" de género, de manera que ante la elección de un candidato, o un sucesor los hombres sienten preferencia por los de su mismo sexo. Estas decisiones derivan en gran medida de los estereotipos de género vinculados al periodista de sexo masculino y muy relacionados con lo que se considera que debe ser un buen líder: individualismo, ambición, eficacia, agresividad, frialdad....

Este corporativismo se prolonga más allá de la jornada laboral. En las relaciones informales los varones, prefieren compartir los momentos de ocio con otros hombres: salir de copas, tomar cerveza... La impronta cultural y la educación recibida implican cierto estilo de diversión y temas de conversación en los que las mujeres son descartadas. A este aspecto hay que añadir la doble jornada laboral de las mujeres ya que al finalizar su trabajo tienen que hacerse cargo de las tareas del hogar, sin que su pareja sea corresponsable en ese ámbito privado. Esto origina un círculo vicioso en el que los hombres en el poder se perpetúan por esas preferencias de género, lo que hace que el cristal del techo se transforme en cemento para esas mujeres que no cuentan con el apoyo de sus superiores por preferencias de género.

Institucionalmente también se tiene una imagen masculina de la profesión. Según Enrique Aguinaga (2002), en 1994 la Comisión Constitucional del Congreso de los Diputados, llamó a comparecencia a catorce prohombres del Periodismo, de la Empresa, de la Enseñanza y del Derecho para formularles la pregunta que, desde hace muchos años, viene repitiéndose, con escaso resultado, en foros y congresos especializados: ¿Quién es periodista?. Pues bien estos profesionales fueron justamente eso, hombres. Ni una sola mujer apareció en el Congreso para definir la profesión. Pero qué ocurre si, además, las asociaciones profesionales que deben luchar por los derechos de sus trabajadores, reproducen el esquema de las empresas. ¿Quién se encargará de promover la igualdad de género en los medios?

La propia Federación de Asociaciones de Periodistas de España (FAPE) creó un consejo de redacción para su publicación Periodistas, noticia difundida por 233grados el 2 de noviembre de 2010 en la que de los diez nombres que componían dicho consejo sólo dos eran mujeres.

Por supuesto hay leyes, hay instituciones públicas como el Ministerio de Sanidad, Política Social e Igualdad y el Instituto de la Mujer que luchan por conseguir la igualdad. Entre ellas hay que destacar la ley orgánica 3/2007, de 22 de marzo, para la igualdad efectiva de mujeres y hombres. O el Parlamento Europeo y el Consejo de la Unión Europea con la Directiva 2002/73/CE que modifica una la directiva previa 76/2007/CEE relativa a la aplicación del principio de igualdad de trato entre hombres y mujeres en lo que se refiere al acceso al empleo, a la formación y a la promoción profesionales, y a las condiciones de trabajo. Pero se trata de una igualdad formal que todavía está muy lejos de la real. 
En este artículo queremos hacer un recorrido por las asociaciones encargadas de velar por los derechos de la profesión de periodista y conocer en qué medida se reproducen los mismos patrones que en las empresas. Todo esto en el marco de una profesión que se está feminizando, el $70 \%$ de los matriculados en Periodismo son mujeres, y ya son mayoría en las redacciones de los periódicos.

Según el Informe Anual de la Profesión Periodística de 2009, editado por la Asociación de la Prensa de Madrid (pp.107-126) desde que se iniciaron los estudios de periodismo en 1972 más de 69.000 licenciados han egresado de las aulas de los 36 centros en los que se imparte el nuevo grado o la licenciatura. Este año se han incorporado 2.500 nuevos licenciados al mercado laboral y los alumnos matriculados en dichos estudios han pasado de 29.895 en el curso 1995-1996 a 48.899 en el curso 2008-2009. El porcentaje de mujeres egresadas ha sufrido muy poca variación en 16 años, ya que ha pasado de un $64,4 \%$ en 1994 a un $69,6 \%$ en 2009 , apenas cinco puntos.

Gráfico 1. Porcentaje de mujeres licenciadas en periodismo (1994-2009)

\% Mujeres licenciadas en Periodismo

(1994-2009)

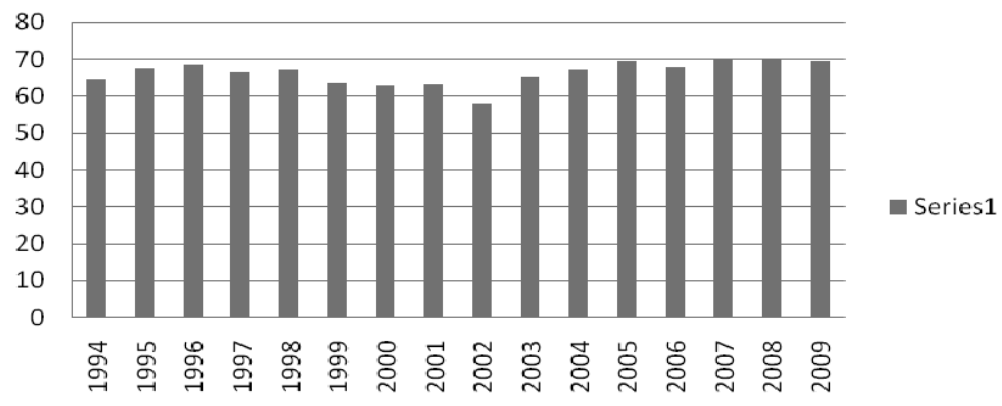

Fuente: Encuestas de la APM facultades, septiembre 2009. INE 2008. Consejo de Coordinación Universitaria 2009 (Publicado en Informe Anual de la Profesión Periodística 2009)

Según Rivero SANTAMaría (2010) en un estudio realizado sobre los estudiantes de la Universidad del País Vasco: "Los porcentajes muestran con claridad que en los últimos diez años un $67 \%$ de las personas que concluyeron sus estudios de periodismo en la UPV-EHU han sido mujeres frente al 33\% de hombres. De manera que podemos establecer sin lugar a dudas que los estudios de periodismo están claramente feminizados, más aún si encajamos este dato dentro de la horquilla del $31 \%$ al $42 \%$ de mujeres que optan por cursar estudios "sociales" en la UPV-EHU. En definitiva, la carrera de periodismo supera en 25 puntos la media de mujeres que lo cursan en comparación con otras carreras catalogadas como "sociales" y en las que la propia universidad ya nos advertía de una feminización de las mismas".

Pero no se trata únicamente de presencia, según los estudios realizados por esta autora, las mujeres además realizan un aprovechamiento superior de la misma y sus calificaciones son también más altas, "(Rivero, 2010) de cada uno de los cursos académicos se han ex- 
traído los quince mejores resultados en función de sexo. Del total de los ciento cincuenta alumnos/as que componen este ranking un $73 \%$ son mujeres y un $27 \%$, hombres. Con estos porcentajes en la mano parece confirmarse la hipótesis de que las mujeres obtienen un mayor aprovechamiento y resultados de su paso por la universidad".

Viendo el gráfico anterior, podría afirmarse que los estudios de periodismo tienen un marcado carácter femenino desde hace al menos tres lustros, lo que justificaría que las licenciadas de entonces, ahora rondando la cuarentena, se encontrarán en un porcentaje similar entre los cuadros directivos medio/alto de los distintos medios de comunicación y, por consiguiente, en las asociaciones en las que se agrupan los profesionales.

\section{EI asociacionismo profesional en el periodismo}

Frente al poder que tiene la patronal en las empresas, los trabajadores han ido agrupándose para defender sus oficios o profesiones. Como indica FERNÁNDEZ (2001), ya en la década de los años treinta, CARR-SAUNDERS y WiLSON (1933) establecieron algunas motivaciones que llevan a las personas a formar agrupaciones profesionales:

a) la definición de una línea de demarcación entre personas cualificadas y no cualificadas;

b) el mantenimiento de altos niveles de carácter profesional o práctica honorable;

c) la elevación del estatus del grupo profesional; y

d) el deseo de la profesión de ser reconocida por la sociedad como la única plenamente competente para realizar su tarea particular.

Las asociaciones profesionales deben por tanto velar por los intereses de la profesión, impulsar la legislación que la normalice, promover el desarrollo de sus miembros y especificar quién puede o no ejercerla, entre otras actividades (FERNÁNDEZ, 2001) (GUILLÉN, 1990).

Estas agrupaciones profesionales comenzaron a aparecer en Europa y América a principios del siglo XIX, y se generalizaron en el período 1880-1920. Es en estás décadas cuando surgen los primeros movimientos asociativos en el sector de la prensa en España. Va emergiendo entre los periodistas la inquietud por definir y agrupar a los profesionales de la información con el objetivo de evitar el intrusismo. Pero, históricamente, los directores de los periódicos han sido capaces, pese a las rivalidades de sus cabeceras, de ponerse de acuerdo para hacer frente a las reivindicaciones sociales de este colectivo.

El 29 de diciembre de 1888, El Imparcial de Madrid publicaba la siguiente información:

"Con motivo de las frecuentes usurpaciones que del nombre de periodistas hacen algunos vividores, se ha pensado en agremiar, o mejor dicho, en dar carácter colegiado a la profesión para que el escritor consagrado a ilustrar la opinión no se le confunda con cualquiera que se pueda presentar con tal título, para que pueda disfrutar de las debidas ventajas que hoy son negadas por miedo a tales confusiones, entendemos que sería cosa hacedera formar un Sindicato de la Prensa y abrir un verdadero registro donde queden inscrito los nombres de los sujetos consagrados a la mencionada profesión." 
Ésta tarea no era nada fácil dadas (ya entonces) las circunstancias precarias en las que trabajaban los periodistas. En su Historia de la Asociación de la Prensa de Cádiz (1909-1992), Fátima SALAVERRY BARO escribe:

"Los bajos salarios provocaron en la práctica que los periodistas alternaran su labor en el periódico con otras labores profesionales, sobre todo en aquellas relacionadas con un organismo oficial, que llevaba una mayor estabilidad profesional. Este es el caso en el que se vieron a numerosos periodistas de Cádiz en el año 1909, algunos, por cierto, fueron los primeros promotores y adheridos a los trabajos de constitución de la Asociación de la Prensa de Cádiz" (SAlaverry, 1993)

Los primeros profesionales en agruparse fueron los tipógrafos. Se constituyeron como los primeros sindicalistas y dirigentes del movimiento obrero, entre otras razones porque su nivel cultural era netamente superior al de cualquier otro empleado por cuenta ajena de su tiempo.

La primera asociación de periodistas fue la Liga de la Prensa Malagueña en 1877, que se transformó en Asociación siete años después. Otras asociaciones que se crearon antes del fin del siglo XIX fueron las de Alicante (19 -XI-1882), Valladolid (X11886), Barcelona (1888), Madrid (15-1 1-1895) y Valencia (8 -1-1899)

La primera agrupación profesional a nivel nacional se constituyó en Santander en 1922 tras la convocatoria de una asamblea constituyente por parte de la Asociación de la Prensa de esa ciudad. Se trata de la Federación de Asociaciones de Periodistas en España (FAPE).

Desde el primer momento esta agrupación resalta el carácter benéfico y asistencial de la misma, siguiendo la pauta de las propias entidades que la integran y que, básicamente, tratan de paliar las deficientes condiciones socio-laborales y económicas en que desenvuelven su actividad los periodistas.

Durante el franquismo, la FAPE se convirtió en un ente semioficial. En 1984 se celebró, también en Santander y a instancias de la Asociación de la Prensa de Cantabria, la XLIV Asamblea General que fue llamada de la "refundación".

La Federación de Asociaciones de Periodistas de España es, actualmente, la entidad más representativa del periodismo español. Agrupa a 45 Asociaciones de la Prensa con 12.500 miembros y tres Asociaciones sectoriales de periodistas, que suman otros 3.000 asociados.

El carácter profesional de las asociaciones de la prensa no ofrece la capacidad de defender a sus asociados laboralmente de las empresas ni de negociar un convenio, por esta razón a finales del siglo XX y principio del XXI se crean en España los primeros sindicatos de periodistas, pero este asunto quedará para otra ocasión.

Las Asociaciones federadas en la actualidad son: Albacete, Alicante, Almería, Aragón, Ávila, Badajoz, Burgos, Cáceres, Cádiz, Campo de Gibraltar, Cantabria, Castellón, Ceuta, Ciudad Real, Córdoba, Cuenca, Granada, Guadalajara, Huelva, Jaén, Jerez de la Frontera, La Coruña, La Rioja, Lanzarote-Fuerteventura, Las Palmas, León, Lugo, Madrid, Málaga, Melilla, Mérida, Murcia, Oviedo, País Vasco, Pamplona, Santa Cruz de Tenerife, Santiago de Compostela, Segovia, Sevilla, Soria, Talavera de la Reina, Toledo, Valladolid, Zamora y Unió de Periodistes Valencians. 
Según un estudio realizado por GonZÁLEZ y PANIAGUA (2008) para analizar la situación actual de las asociaciones de periodistas en España, se ha producido un "aumento de la representación femenina, incremento de los servicios ofrecidos, mayor visibilidad en la Red y puesta en marcha de nuevos proyectos". En el censo de la

Gráfico 2. Número de hombres y mujeres asociados

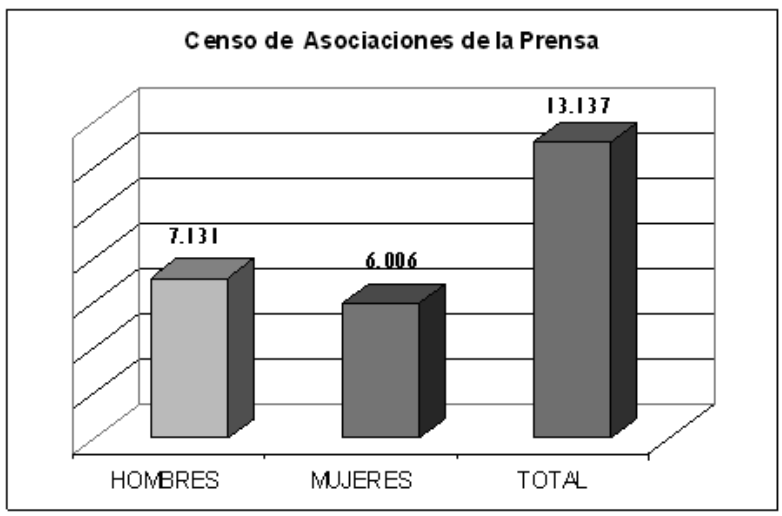

Fuente: FAPE. González y Paniagua (2008) FAPE mostrado por estos autores comprobamos que son muy pocas las asociaciones de periodistas en las que el número de asociadas es superior al de asociados. Destacan la de Aragón, Cáceres, Guadalajara, La Rioja, León, Lugo, Mérida y Talavera de la Reina. En varias de ellas el desfase entre géneros es casi insignificante, sin embargo en otras como las dos primeras es muy significativo. Más adelante estudiaremos si esto repercute en los cargos de dirección o en la composición de las Juntas Directivas.

Continúan estos autores señalando que si se atiende al incremento por sexos, otra tendencia se corrobora en 2007: el mayor porcentaje de mujeres entre los recién incorporados, que significan ya el $45.7 \%$ de los miembros frente al 54.3 por ciento de hombres. En los dos últimos años, aproximadamente un $60 \%$ de los nuevos asociados fueron mujeres, dato que puede ser interpretado como una consecuencia evidente de la mayor tasa de mujeres matriculadas y egresadas de los estudios de periodismo.

En 1994 gracias al desarrollo de una mayor concienciación en la sociedad con los temas de género se crea AMECO (Asociación Española de Mujeres Profesionales de los Medios de Comunicación, www.nodo50.org /ameco) constituida por mujeres profesionales de distintos medios (prensa, radio y televisión, agencias, gabinetes de comunicación, etc.). La misión declarada en su página web es la de "ser un foro plural de opiniones y experiencias, tratando de positivar la imagen de la mujer, tan deteriorada y maltratada, en los medios escritos y audiovisuales"

Gráfico 3. Evolución del censo de la FAPE

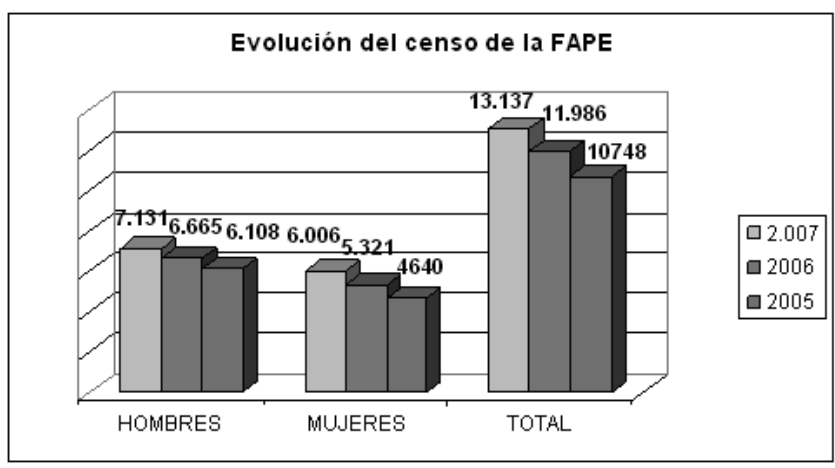

Fuente: FAPE. González y Paniagua (2008) 
Los objetivos de esta asociación de mujeres son: incrementar el protagonismo e influencia de las mujeres en la sociedad a través de los medios de comunicación; promover una imagen real y positiva de la mujer en los medios; defender la igualdad de oportunidades en el acceso a los puestos de dirección y decisión en los medios; desarrollar y potenciar la carrera de las mujeres profesionales en los medios en su actividad laboral; organizar encuentros, jornadas y elaborar estudios y establecer vínculos con otras asociaciones, tanto nacionales como internacionales.

Siguiendo con esta línea ha de incluirse una breve mención a la escasez de observatorios que funcionan entre las Asociaciones. Sin embargo, de los tres existentes dos están relacionados con temas de género: el de la Asociación de la Prensa de Cádiz de las mujeres y el de la agrupación malagueña sobre violencia de género.

\section{Objetivos}

El objetivo de esta investigación es analizar la estructura directiva de las asociaciones profesionales de periodistas desde la óptica de género, partiendo del supuesto de que las organizaciones en las que la representación de mujeres es baja o que son dirigidas por hombres mostrarán menos interés por las cuestiones de igualdad en la profesión y alimentarán la perpetuación de los patrones actuales en las empresas periodísticas.

Estos objetivos los podemos exponer en las siguientes hipótesis que trataremos de contrastar:

H1. Las mujeres están subrepresentadas en las Juntas Directivas de las asociaciones profesionales de periodistas.

H2. Existen pocas mujeres presidiendo asociaciones profesionales

H3. Las asociaciones profesionales presididas por mujeres cuentan con un mayor número de mujeres en los cargos de gestión de la misma.

H4. Las asociaciones con mayoría de mujeres asociadas cuentan con más mujeres en las juntas directivas.

\section{Metodología}

Hemos buscado la información en el listado de asociaciones publicado por la FAPE (Federación de Asociaciones de la Prensa), en dicho listado aparecen enlaces a las diferentes asociaciones provinciales. Del total de 47 asociaciones solo hemos encontrado datos de 32 (el 68 por ciento), al carecer el resto de página web, o no indicar en su página la composición de la junta directiva. El número de registros (miembros de las juntas directivas) ha sido de 321. Los datos fueron recopilados en julio de 2010.

Hemos elaborado una hoja de cálculo donde analizamos el número de hombres y mujeres que componen las Juntas Directiva e identificamos el sexo de la persona que ocupa la presidencia.

Los datos han sido tratados utilizando operaciones estadísticas simples que pongan de manifiesto las desigualdades por sexo que existe en estas organizaciones que representan al colectivo profesional de los periodistas.

\section{Resultados}

Hemos analizado la presencia de mujeres en las Juntas Directivas de las Asociaciones profesionales del periodismo, para comprobar que al igual que ocurre en las audien- 
cias, o en los puestos directivos, las mujeres están subrepresentadas en estas organizaciones encargadas de luchar por los derechos de los profesionales del sector.

En el total de Juntas Directivas aparecen 321 personas, de las cuales 165 son hombres y 156 mujeres.

Gráfico 4. Porcentaje de mujeres y hombres presentes en diferentes organizaciones.

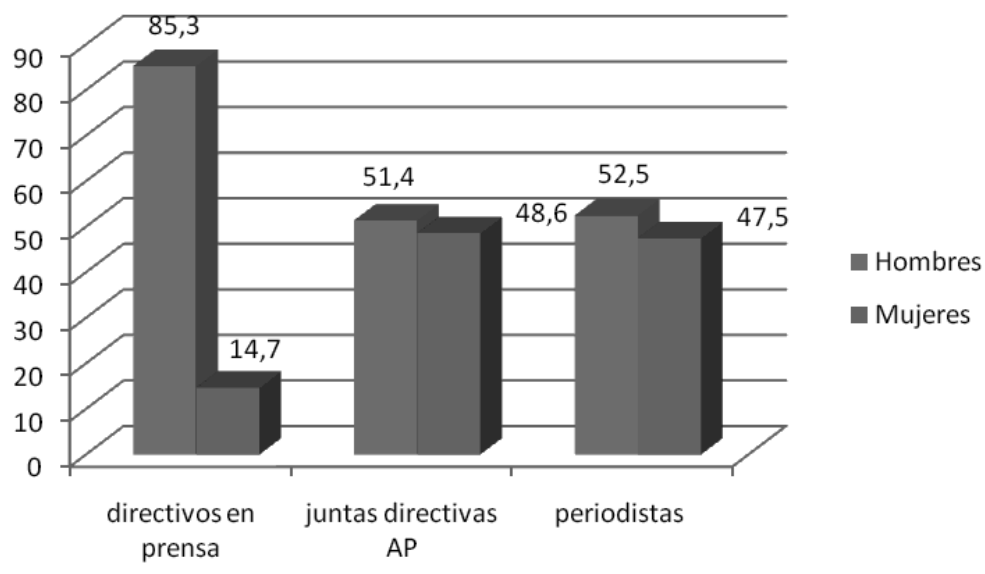

Fuente: elaboración propia

La proporción del $48.6 \%$ de mujeres y el $51.4 \%$ de hombres es aceptable y refleja casi con exactitud la realidad de la profesión (52,5\% hombres y $47,5 \%$ de mujeres). Si comparamos el dato con el de mujeres con cargos directivos en las redacciones de prensa se constata como en las asociaciones la igualdad entre los sexos está mejor reflejada. En los diarios el 85,3\% de los cargos directivos (director, jefes de redacción y de sección) son hombres frente a tan solo un 14,7\% mujeres.

Sin embargo, al analizar los datos incorporando otros criterios podemos percibir como también en las asociaciones profesionales se reproducen los patrones desfavorables para las mujeres y que de ninguna manera favorecen el desarrollo de medidas de igualdad en la profesión.

De las 32 asociaciones profesionales estudiadas, 17 tienen más hombres que mujeres en su composición, 12 más mujeres y 3 tienen el mismo número. Es decir, en el $53 \%$ de las organizaciones los hombres son mayoría frente a un 38\% con mayoría de mujeres.

Si atendemos al número de mujeres presidiendo las asociaciones encontramos una subrepresentación en los puestos máxima responsabilidad y más visibilidad, tan sólo asumen este cargo 11 mujeres frente a 21 hombres, es decir, un 34\% frente a un $66 \%$. En este caso la proporción no es representativa de las mujeres que están presentes en las Juntas Directivas que hemos visto que prácticamente es paritaria.

En cualquier caso, si lo comparamos con el porcentaje de mujeres directoras de periódicos el dato es muy positivo, $9,6 \%$ de mujeres frente al $90,4 \%$ de hombres. 
Al considerar el porcentaje de mujeres en la profesión frente al de hombres $(47,5 \%$ frente al 52,5\%), llama la atención esa diferencia porcentual de más de un $20 \%$, sobre todo teniendo en cuenta que nos encontramos en organizaciones democráticas que no están sometidas a las decisiones de los empresarios que en su mayoría son hombres. Este dato sigue poniendo de manifiesto que existen barreras para las mujeres más allá de las limitaciones culturales y los patrones

Gráfico 5. Porcentaje de mujeres y hombres en las juntas directivas de las AP según el sexo de la persona que ocupa la presidencia

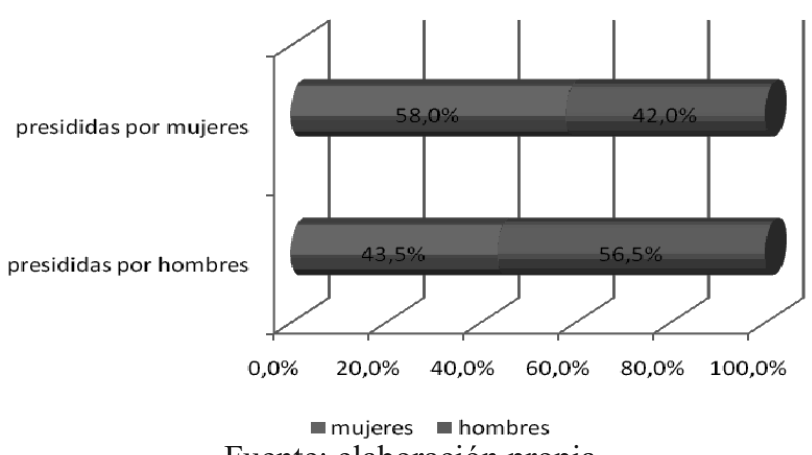

Fuente: elaboración propia

Un dato interesante es conocer si las asociaciones presididas por mujeres cuentan con más presencia de personas de sexo femenino.

En el gráfico 5 se aprecia como las asociaciones presididas por mujeres cuentan con un $58 \%$ de mujeres en la Junta Directiva frente a un $43.5 \%$ en las empresas presididas por hombres. Se evidencia la preferencia de las mujeres presidentas para trabajar con otras mujeres al igual que los hombres prefieren rodearse de otros hombres cuando ocupan puestos de dirección. Se da un fenómeno que podríamos denominar "complicidad de sexos"

También es llamativo que las asociaciones que tienen mayor presencia de mujeres (mm) que de hombres ( $\mathrm{mh})$, están en su mayoría presididas por mujeres (7 mujeres frente a 5 hombres). En el caso de las que tienen mayoría de hombres nos encontramos con que sólo hay 3 mujeres frente a 14 hombres. Se vuelve a poner de manifiesto la relación entre mujeres directivas y el sexo de las personas a su cargo.

Gráfico 6. Número de asociaciones con mayoría de mujeres u hombres según el sexo del presidente

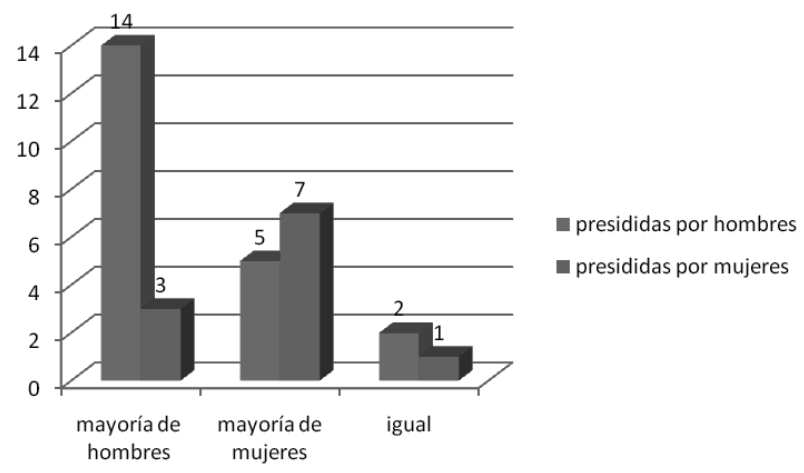

Fuente: elaboración propia 
Si establecemos un ranking según la presencia de mujeres en las asociaciones, apreciamos que entre las ocho primeras (con mayor porcentaje de mujeres) tan sólo hay dos presididas por hombres.

En el extremo opuesto, en las 8 en las que hay menos mujeres en la junta directiva, todas están presididas por hombres.

Nuevamente se vuelve a confirmar el peso de la complicidad de género a la hora de elegir el sexo de las personas con las que se quiere trabajar.

Tabla 1. Ranking de asociaciones según el porcentaje de mujeres en la junta directiva

\begin{tabular}{|c|c|c|c|}
\hline$\overline{\text { RANK }}$ & ASOCIACIÓN & Preside & $\% \mathrm{~m}$ \\
\hline 1 & Asociación de la Prensa de Burgos & $\mathrm{M}$ & $83,3 \%$ \\
\hline 2 & Asociación de la Prensa de Toledo & $\overline{\mathrm{H}}$ & $80,0 \%$ \\
\hline 3 & Asociación de la Prensa de Jerez & $\mathrm{M}$ & $75,0 \%$ \\
\hline 4 & Asociación de la Prensa de Cantabria & $\bar{M}$ & $70,0 \%$ \\
\hline 5 & Asociación de Vigo & $\mathrm{M}$ & $66,7 \%$ \\
\hline 6 & Asociación de la Prensa de Aragón & $\mathrm{M}$ & $63,6 \%$ \\
\hline 7 & Asociación de Periodistas de Albacete & $\mathrm{M}$ & $60,0 \%$ \\
\hline 8 & Asociación de la Prensa de La Rioja & $\mathrm{H}$ & $60,0 \%$ \\
\hline 9 & Asociación de la Prensa de Valladolid & $\mathrm{H}$ & $60,0 \%$ \\
\hline 10 & Asociación de la Prensa de Lugo & $\mathrm{M}$ & $55,6 \%$ \\
\hline 11 & Asociación de la Prensa de Cataluña & $\mathrm{H}$ & $54,5 \%$ \\
\hline 12 & Asociación de la Prensa De Granada & $\mathrm{H}$ & $54,5 \%$ \\
\hline 13 & Asociación de la Prensa de Cádiz & $\mathrm{H}$ & $50,0 \%$ \\
\hline 14 & Asociación de la Prensa de Huelva & $\bar{M}$ & $50,0 \%$ \\
\hline 15 & Asociación Salmantina de Periodistas & $\mathrm{H}$ & $50,0 \%$ \\
\hline 16 & Asociación de la Prensa de Sevilla & $\mathrm{M}$ & $47,1 \%$ \\
\hline 17 & Asociación de la Prensa de Murcia & $\mathrm{H}$ & $46,7 \%$ \\
\hline 18 & Asociación de la Prensa de Santa Cruz de Tenerife & $\mathrm{H}$ & $45,5 \%$ \\
\hline 19 & Asociación de la Prensa de Madrid & $\mathrm{H}$ & $45,0 \%$ \\
\hline 20 & Asociación de la Prensa de Cáceres & $\bar{M}$ & $44,4 \%$ \\
\hline 21 & Asociación de la Prensa de Guadalajara & $\mathrm{H}$ & $42,9 \%$ \\
\hline 22 & Unió de Periodistes Valencians & $\mathrm{H}$ & $42,9 \%$ \\
\hline 23 & Asociación de la Prensa de Almería & $\mathrm{M}$ & $41,7 \%$ \\
\hline 24 & Asociación de la Prensa de Málaga & $\mathrm{H}$ & $41,7 \%$ \\
\hline 25 & Asociación de la Prensa de Jaén & $\mathrm{H}$ & $40,0 \%$ \\
\hline 26 & Asociación de la Prensa de Mérida & $\mathrm{H}$ & $33,3 \%$ \\
\hline 27 & Asociación de la Prensa de Alicante & $\mathrm{H}$ & $33,3 \%$ \\
\hline 28 & Asociación de la Prensa de La Coruña & $\mathrm{H}$ & $30,0 \%$ \\
\hline 29 & Asociación de la Prensa de Oviedo & $\mathrm{H}$ & $25,0 \%$ \\
\hline 30 & Asociación de la Prensa de Santiago de Compostela & $\mathrm{H}$ & $22,2 \%$ \\
\hline 31 & Asociación de la Profesionales de la Información de Soria & $\mathrm{H}$ & $20,0 \%$ \\
\hline \multirow[t]{2}{*}{32} & Asociación de la Prensa de Navarra & $\mathrm{H}$ & $14,3 \%$ \\
\hline & Totales & & $48,6 \%$ \\
\hline
\end{tabular}




\section{Conclusiones}

Las asociaciones profesionales tienen un papel muy importante en la defensa de la profesión, no solo en cuanto a qué personas pueden ejercerla, si no también en cuanto a la calidad del trabajo que ofrecen. En los últimos años, estas agrupaciones están tratando de desarrollar un papel más activo respecto a las condiciones laborales en las que los periodistas ejercen su trabajo. Luchan contra la precariedad, el desprestigio y la desigualdad en la profesión.

Partíamos del supuesto de que si estas asociaciones estuvieran constituidas en sus órganos de gobierno de manera igualitaria los esfuerzos que dedicarían a desterrar las desigualdades de género en la profesión serían mayores que en el caso de que se reprodujeran los patrones de las empresas periodísticas con gestores y directivos de sexo masculino.

Hemos constatado que las diferencias con respecto a las empresas son considerables cuantitativamente, pero al mismo tiempo comprobamos que algunas de las barreras que dificultan el acceso de la mujer a los puestos de toma de decisiones siguen perpetuándose. Hay pocas mujeres presidiendo estas asociaciones y cuando son presididas por hombres se percibe favoritismo por la presencia de hombres en sus Juntas Directivas.

Veamos la contrastación de hipótesis planteadas al inicio de la comunicación:

H1. Las mujeres están subrepresentadas en las Juntas Directivas de las asociaciones profesionales de periodistas. Hemos constatado como esta hipótesis no se corrobora, la proporción de hombres y mujeres en las Juntas Directivas de estas asociaciones es muy próxima a la paridad y refleja casi con exactitud el porcentaje de hombres y mujeres en la profesión.

H2. Existen pocas mujeres presidiendo asociaciones profesionales. El porcentaje de mujeres que presiden las asociaciones de profesionales es muy inferior al de hombres. Los hombres siguen copando los puestos clave de las organizaciones independientemente de su naturaleza pública, privada y de sus fines (lucrativas o no lucrativas)

H3. Las asociaciones profesionales presididas por mujeres cuentan con un mayor número de mujeres en los cargos de gestión de la misma. Se constata la existencia de un corporativismo de género. Las presidentas se rodean de mujeres en sus juntas directivas y de la misma manera los hombres se rodean de hombres. Esta tendencia es negativa ya que al existir mayoría de hombres presidiendo estas asociaciones se puede perpetuar el hábito y aparecer un techo de cristal que dificulta el acceso de las mujeres.

H4. Las asociaciones con mayoría de mujeres asociadas cuentan con más mujeres en las juntas directivas. No se constata esta hipótesis. No hemos encontrado una relación directa entre el número de mujeres asociadas y la presencia de mujeres directivas.

Queda por tanto aún mucho camino por recorrer en la lucha por la igualdad dentro de la profesión. Las asociaciones profesionales deben jugar un papel relevante 
estos temas y para ello es preciso un mayor esfuerzo en esta dirección. La incorporación de las mujeres a las Juntas Directivas puede contribuir a un mayor desarrollo de medidas de igualdad en la profesión al incluir a las "afectadas" en los órganos competentes para ejercer presión ante las empresas e instituciones ligadas con el periodismo.

En este sentido, el desarrollo de políticas igualitarias en las asociaciones contribuirá a que la profesión refleje mejor la situación actual de las mujeres en este sector tan relevante para generar opinión y ciudadanos críticos.

Para finalizar la reciente recomendación de la FIP a sus asociados, que "las organizaciones formen sus propias comisiones de género" e insiste en su transversalidad de manera que la "cuestión de género no sea algo aislado, sino que esté presente en toda la actividad de la organización internacional de periodistas y que se cuente con la participación no solamente de las mujeres sino también de los hombres" (SAMPERIO, 2011: 38).

\section{Referencias Bibliográficas}

AGUINAGA, Enrique de (2002): "El periodista en el umbral del siglo XXI". Estudios sobre el mensaje periodístico, vol. 8, pp. 157-170

BURKE, Ronald J. y MATTHIESEN, Stig Berge (2009): "Workaholism among Norwegian journalists: gender differences". Equal Opportunities International, vol. $28, \mathrm{n}^{\circ} 6$.

CARO, Francisco J. y GARCÍA, María del Mar (2009): "Las mujeres directivas en las empresas periodísticas. Una aproximación desde la Grounded Theory". Identidades Femeninas en un Mundo Plural, págs. 125-130.

CARR-SAUNDERS, Alexander Morris and WILSON, Paul Alexander (1933): The Professionals. London, Frank Cass.

DJERF-PIERRE, Monika (2007): "The Gender of Journalis. The Structure and Logic of the field in the Twentieth Century". Nordicom Review, pp. 81-104.

FARIAS BATLLE, Pedro (director, 2009): Informe anual de la profesión Periodística 2009. Madrid, Asociación de la Prensa de Madrid.

FRÖLICH, Romy y LUDWIN, Maximilians (2007): "Three steps forward and two back?" en Women in Mass Comunication. California, Sage Publications.

FERNÁNDEZ PÉREZ, Jorge (2001): "Elementos que consolidan el concepto de profesión. Notas para su reflexión". Revista Electrónica de Investigación Educativa, 3 (2). Consultado el 5 de enero de 2010 en: http://redie.uabc.mx/vol3no2/contenido-fernandez.html

GONZÁLEZ, María Eugenia y PANIAGUA, Francisco Javier (2008): "Las asociaciones de la prensa españolas, un modelo de organización profesional consolidado". Revista Latina de Comunicación Social, 63, páginas 435 a 444. La Laguna (Tenerife), Universidad de La Laguna, recuperado el 11 de 01 de 2011, de http://www.revistalatinacs.org/08/36_793_56_prensa/Gonzalez_y_Paniagua.html 
GUILLÉN, Mauro F. (1990): "Profesionales y Burocracia: Desprofesionalizacion, Proletarizacion y Poder Profesional en las Organizaciones Complejas". Reis. 51/90, pp. 35-51.

RIVERO SANTAMARINA, Diana (2010): "La feminización de los estudios de periodismo: análisis del caso de la UPV/EHU". II Jornadas Mujer y Medios de Comunicación, pp. 85-104.

SALAVERRY BARO, Fátima (1993): Historia de la asociación de la Prensa de Cádiz 1909-1992. Cádiz, Asociación de la Prensa.

SAMPERIO, María Ángeles (2011): "El Comité de Género de la FIP debate su Funcionamiento". Periodistas, $\mathrm{n}^{\circ} 23$.

SORIANO, Jaume; CANTÓN, María José; y DÍEZ, Mercé (2005): "La pseudofeminización de la profesión periodística en España”. Zer, n 19, pp. 35-52. 